MODELING, IDENTIFICATION AND CONTROL, 2005, VOL. 26, No. 4, 221-235

doi:10.4173/mic.2005.4.3

\title{
Advanced life support therapy on out-of-hospital cardiac arrest patients: Applying signal processing and pattern recognition methods
}

\author{
TRYGVE EFTESTØL*, MARTIN RISDAL $\dagger$, JOAR EILEVSTJØNN \\ and PETTER ANDREAS STEENI
}

Keywords: Cardiac arrest, ventricular fibrillation, cardiopulmonary resuscitation, ECG analysis, decision support, defibrillation, CPR artefact removal

In the US alone, several hundred thousands die of sudden cardiac arrests each year. Basic life support defined as chest compressions and ventilations and early defibrillation are the only factors proven to increase the survival of patients with out-of-hospital cardiac arrest, and are key elements in the chain of survival defined by the American Heart Association. The current cardiopulmonary resuscitation guidelines treat all patients the same, but studies show need for more individualization of treatment. This review will focus on ideas on how to strengthen the weak parts of the chain of survival including the ability to measure the effects of therapy, improve time efficiency, and optimize the sequence and quality of the various components of cardiopulmonary resuscitation.

There are several hundred thousand sudden cardiac deaths in the United States each year with an estimated event rate of one sudden cardiac arrest per 1000-person years (Guidelines 2000) and survival rate of less than $10 \%$. Similar dismal numbers are reported in other countries worldwide.

At present only two factors are proven to increase survival of patients with out-of-hospital cardiac arrest: basic life support (BLS) defined as chest compressions and ventilation, and defibrillation of patients with ventricular fibrillation (VF) or pulseless ventricular tachycardia (VT) (Guidelines 2000). The whole purpose of using a defibrillator is to eliminate the chaotic electrical activity of the heart during VF or the too-rapid ventricular activity in VT which precludes a circulation, hoping that the regular rhythm-generating system of the heart again can take over and generate a pulse (circulation of blood). No drugs being used during cardiopulmonary resuscitation (CPR) have been shown to improve clinical outcome (Guidelines 2000). Without the use of a defibrillator no patients with VF obtain return of spontaneous circulation (ROSC). With the use of a defibrillator approximately $40-60$ percent of these patients might achieve ROSC (Hargartem et al., 1990; Wik et al., 2003; Sunde et al., 1999) with a survival rate to hospital discharge varying between 5 and 30\% (Hargartem et al., 1990; Wik et al., 2003; Sunde et al., 1999; Herlitz et al., 1999; Eisenberg et al., 1990). For other cardiac

*University of Stavanger, Department of Electrical and Computer Engineering, N-4036 Stavanger, Norway, e-mail: trygve.eftestol@uis.no

$\dagger$ University of Stavanger, Department of Electrical and Computer Engineering.

+Laerdal Medical AS, P.O. Box 377, N-4002 Stavanger, Norway, e-mail: joar.eilevstjonn@laerdal.no

IUUleval University Hospital. Department of Anesthesiology, N-0407 Oslo, Norway, e-mail: p.a.steen@medisin.uio.no 
rhythms, the prognosis is dismal, with a survival rate of 1-2\% (Eisenberg et al., 1990), and most survivors have gone through a stage with VF and successful defibrillation before achieving ROSC.

Defibrillation has therefore been moved forward in the chain of survival for patients with cardiac arrest, and the new international guidelines state that early defibrillation within 5 minutes of call-receipt by the emergency medical service (EMS) is a high priority goal (Guidelines 2000). To achieve this, non-medical personnel must be enabled to defibrillate in so-called public access defibrillation (PAD) programs using automated external defibrillators (AEDs). Marenco et al reviewed the role of the automated defibrillator in improving survival from sudden cardiac death (Marenco et al., 2001). The focus has turned onto development of more efficient waveforms, and designing lightweight, easy-to use defibrillators.

The following review will be limited to the out-of-hospital setting where AEDs are used primarily for the termination of VF. A short outline of therapy according to the current guidelines of advanced life support (ALS) precedes the discussion of factors weakening the chain of survival. Following this, we discuss suggestions for improvements and possible means to achieve these applying signal processing and pattern recognition methods. This article is modified from a previous review article (Eftestøl et al., 2003). The modifications are minor, mostly presenting an update on recent results.

\section{Current guidelines}

The AED records an ElectroCardioGram (ECG), and uses a software algorithm analyzing the ECG waveforms to determine whether the rhythm is shockable (VF or VT) or not. Based on the analysis, a voice prompt recommends defibrillation or not.

The current CPR guidelines recommend immediate defibrillation if the first recognized rhythm is shockable. If this first shock is unsuccessful in terminating the VF or VT, up to two more shocks are recommended before BLS is started. Thereafter series of defibrillation attempts and BLS periods are repeated until ROSC is achieved or the whole CPR attempt is discontinued. For non-VF/VT rhythms, BLS is continued until a shockable rhythm is achieved, or the whole CPR attempt is discontinued.

\section{Problems with current guidelines-the weak chain}

The most important factors influencing the survival rates of out-of-hospital cardiac arrest patients are:

- Time elapsed from collapse until the first defibrillation attempt (Hargarten et al., 1990; Larsen et al., 1993; Wik et al., 2003).

- Whether BLS has been provided or not (Cummins et al., 1985).

- The quality of both BLS and ALS (Van Hoeywegen et al., 1993; Wik et al., 1995).

The interplay of these factors is decisive for the final outcome. Recent studies strongly indicate that it is time-dependent whether defibrillation should be attempted as soon as a defibrillator is available, if good-quality BLS has not been provided in the meantime. If more than a few minutes have passed from the time of arrest until the defibrillator arrives (more than 4-5 minutes response time), a 1.5-3 minute period of BLS should precede the defibrillation attempt (Cobb et al., 1999; Wik et al., 2003). This is probably due to myocardial deterioration during the arrest period resulting in an 
inability of the regular rhythm-generating system of the heart to take over after a defibrillation attempt.

\section{Preparing for, delivering and determining outcome after the use of defibrillations}

Many studies report very high defibrillation success rates, 95-99\%. Yet the ROSCrate is frequently less than $50 \%$, and many patients achieving ROSC require many defibrillation attempts (Hargarten et al., 1990; Sunde et al., 1999). This might be somewhat confusing, and is due to the definition of defibrillation success. As mentioned above, the immediate purpose of using the defibrillator is to eliminate VF or VT, hoping that this will give room for a pulse-generating rhythm (ROSC). Thus defibrillation success is frequently defined as the absence of VF or VT for at least 5 seconds after the shock (Gliner \& White, 1999). Clinically, we are interested in ROSC which as suggested above does not only depend on the quality of the defibrillator and defibrillation attempt. but also on the condition of the myocardium, which again depends on the duration of the cardiac arrest and the BLS provided (Wik et al., 2003). A clinical definition of a successful defibrillation attempt could therefore be ROSC, stable or not (Sunde et al., 1999). In these terms, only $10 \%$ of 883 shocks were successful in the study by Sunde et al., 1999.

It would be of great potential advantage if defibrillation attempts not resulting in ROSC could be avoided. It has been shown that a defibrillation attempt in itself is harmful to the myocardium.

It would therefore be desirable if the myocardial situation, and thus the probability of ROSC, could be predicted before defibrillation is attempted. There are now quite a few studies showing that the VF waveform contains such information (Noc et al., 1999; Eftestøl et al., 2000; Small et al., 2001; Strohmenger et al., 2001; Povoas et al., 2002). This information could then be used to decide whether BLS should be given first in an attempt to increase probability of ROSC. As discussed in more detail below, this information is represented by features calculated from ECG waveforms, the hypothesis being that the feature characteristics reflects changes in resuscitability.

\section{Aspects of hands-off-intervals (HO)}

We discussed the problem of unsuccessful defibrillations above. In addition to the myocardial damage, it is important to consider that each such defibrillation attempt requires a time period for rhythm analysis, defibrillator charging, shock delivery and new post-shock rhythm analysis where no BLS can be given, as the rhythm analysis software requires that the patient lics completely still to avoid any ECG artefacts being created. In one study, the median time from initiation of rhythm analysis until shock was given, was 20 seconds (Sunde et al., 1999). In a follow-up study of the same material the myocardial condition deteriorated substantially during that same period (Eftestøl et al., 2002). In general one might say that the chance for ROSC is at its highest level immediately after the arrest. when the heart muscle still has a high supply of energy resources and oxygen. As time passes, these resources are drained. The rate of this drainage can at least be reduced by properly performed BLS, which as mentioned above, might even improve the myocardial situation if there has been a period without properly executed BLS before the defibrillator is available. Such an improvement was indeed demonstrated in a study of VF waveform changes due to precordial compressions (Eftestøl et al., 2004). With series of three defibrillation attempts followed by one-minute 
periods of BLS, approximately $50 \%$ of the time is spent without any blood flow being generated to the tissues.

\section{Decision support-identifying and strengthening the weak chains of survival}

Summarizing, some of the crucial problems in resuscitation are:

- A large number of shocks are unsuccessful.

- Much hands-off time, without blood flow being generated, occur with the use of presently available AEDs.

- Quality of compressions and ventilations delivered might be poor.

The last 15 years has seen advances in research addressing these issues, investigating methods that have a potential for improving cardiac arrest survival rates. In essence, the rescuer should be guided by information at least partly gathered on-line and processed in real-time, in her/his decision making on which therapeutic component to use. Such methods are parts of a decision support system.

A decision support system might be considered as representing the knowledge of former therapy. Data representing the documentation of therapy is structured into this knowledge system (Mitra et al., 2002; Kovalerchuk et al., 2000; Fong et al., 2002). This documentation might include patient demographics, ECG derived information and outcome information. Further analysis techniques are used to find the functional link between patient data and outcome variables. Considering these factors, we might envisage a potential for increased survival if we are able to develop a decision support system incorporating functionality for:

- Reading and integrating multiple sources of information

- Measuring the effects of therapy-online monitoring

- Reducing duration of hands-off-time during ALS and BLS

- Optimize the sequence and quality of the various ALS and BLS components.

Several research groups have been occupied with developing methods pointing toward such a decision support system. We review how advanced information technology methods such as signal analysis and pattern recognition can be used to develop such functionality.

\section{Reading and integrating multiple sources of information}

The therapy of cardiac arrest is documented in several ways:

1. The defibrillator provides an event report of and time series recordings of ECG and in some cases impedance measurements. The coded information appears in a file or several files which might be transmitted to a computer.

2. Report sheets carry information about patient demographics and important therapy, which can been stored electronically.

To extract meaningful information, the data have to be imported and integrated in a software environment. This requires knowledge about coding formats of the event records, ECG and other physiological data (Värri et al., 2001). The data are decoded so that each time series is available as a continuous sequence of numbers on which logical and arithmetic operations can be performed for statistics and signal analysis. Both defibrillator event records and report data are synchronized to the time series data. 


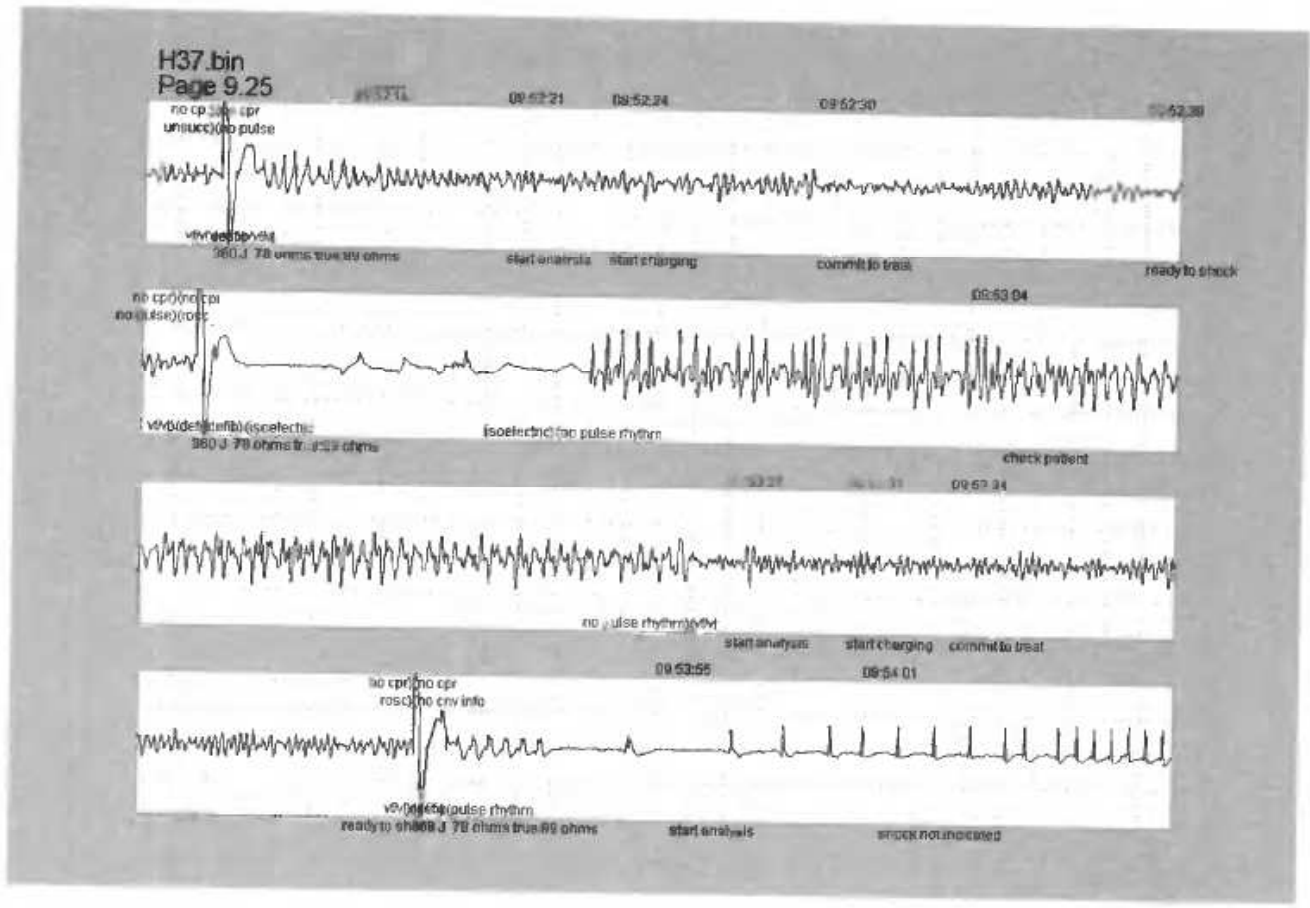

Figure 1: ECG tracing illustrating VF, with artefacts from precordial chest compressions, hands-off intervals, countershocks and return of spontaneous circulation. Each line shows 30 seconds of ECG continuing from the previous line. The time stamps and defibrillator usage text above and below each line of tracing are read from the electronic defibrillator log file. The text within the tracing axes are the therapy, defibrillation outcome and rhythm annotations provided by the medical experts.

Medical experts can then interpret and annotate the data based on integration of this information. Such primary annotations will typically be rhythm changes. From these primary annotations, secondary annotations can be generated automatically. As an example, an algorithm for determining individual defibrillations outcomes can be devised based on which post-shock rhythms are present. Figure 1 illustrates such visualization with the rhythm annotations and CPR sequences identified. The ECG time series is shown as a continuous tracing. The events logged are shown with event descriptions below and time stamps above each axis window aligned with the ECG according to time of appearance. Both primary and secondary annotations are shown within the axis windows with the top ones indicating presence of CPR and the bottom ones showing rhythm changes. The annotations in the second line are examples of secondary annotations indicating the outcome of the next defibrillation.

In recent years, several studies utilizing data from AEDs have given information on exact numbers of shocks given, time intervals between important events, outcomes like conversions after defibrillation attempts, duration of CPR sequences and duration of hands-off intervals (Sunde et al., 1999; Gliner \& White, 1999). For many ycars, statistical information derived from analysis of digitally stored report sheets has also provided valuable information about time intervals such as duration from collapse to call, arrival of ambulance personnel, and delivery of first shock. There are many examples of how such analyses have identified weaknesses in the chain of survival. For instance, in Oslo a lack of decentralization of the ambulances caused long response times (Sunde et al., 2001). Also, identifying and computing hands-off interval duration revealed a 
median time of 20 seconds for preparing for and delivering a shock in the same material (Sunde et al., 1999). Identifying such weaknesses provides opportunities to strengthen the chain, for example by decentralizing the ambulance service, or switching from an AED mode to manual defibrillation, thereby reducing the time spent on rhythm analysis and enabling BLS during defibrillator charging, consequently shortening the duration of the hands-off intervals.

\section{Measuring the effects of therapy-online monitoring}

Work aiming at measuring the effects of therapy goes back 15 years with several rescarch groups involved as reviewed by Amann et al., 2001. In our opinion, the ability to predict the outcome of defibrillation is the key to improve resuscitability. It is the most mature method we will discuss, although no AED available has this kind of functionality. Defibrillation outcome prediction has only been used for retrospective analysis, but is considered in the evidence evaluation and science review process in preparation of the new resuscitation guidelines scheduled for 2006.

The hypothesis is that features can be derived from the ECG waveform. Features with good discriminative power can be used to develop a defibrillation outcome predictor that will enable the avoidance of unsuccessful defibrillation attempts (NO ROSC). Shocks will only be given when the chance for success (ROSC) is high. Such a decision should be based on a compound analysis of one or several features, with the predictor's output being a hard decision SHOCK/DO NOT SHOCK or a soft decision producing a continuous outcome variable. Such a continuous variable can be used to represent the probability of ROSC. An outcome predictor can be developed using several possible methods. We will discuss the basic aspects and give some examples from our own research which applies signal processing and pattern recognition methods.

From a signal processing perspective, the main approaches applied in characterising the VF fall within the categories of time- and frequency domain techniques. It is important to note that the physiological processes related to cardiac arrest change due to time and therapy. Thus, the parameters describing these processes change accordingly. The VF analysis features estimate these parameters which are subsequently used to distinguish VF with a high probability of ROSC from those of low probability. The time domain techniques involve characterisation of the signal's amplitude behaviour. The $\mathrm{VF}$ signal, typically does not exhibit one distinct periodic component, but rather a composite spectrum of several such components which are more naturally expressed in frequency rather than time units. Due to this, and that signals with such properties are commonly and naturally analysed in the frequency domain, the power spectral density (PSD) function has been widely used in the analysis of the signal's periodic behaviour.

Pattern recognition methodology is applied to develop and evaluate a defibrillation outcome predictor from a specific feature combination derived through time- and frequency domain techniques (Duda et al., 2001). In Bayesian decision theory, a pattern represented by a certain feature combination (feature vector) is classified according to a decision rule. This rule is established through estimates of the á priori probabilities of the successful and unsuccessful outcome classes and the class specific probability density functions (PDFs) describing the distribution of the feature vectors. The á priori probability represents our degree certainty of which class the next pattern to be observed belongs to. After observing this pattern, we apply Bayes rule to the á priori probabilities and the class specific PDFs to adjust this certainty into á posteriori probabilities. According to Bayes decision theory, selecting the class according to the highest 
á posteriori probability corresponds to the minimum error decision rule. In some cases, wrong classification of one class of patterns is considered more serious than misclassifications of patterns from the other class. In this case, a weighting of the PDFs can be applied in a risk minimising strategy. In outcome prediction this can be used to prioritise correct classification of successful defibrillations. This ensures that the patient do not receive worse treatment than what is advised by the standard treatment protocol. Amman et al give an excellent overview of feature extraction techniques having been applied in VF analysis (Amann et al., 2001).

The starting point of defibrillation outcome prediction as we defined it in our study of 87 ROSC and 781 NO ROSC VF segments, was to extract VF waveforms and outcome information from the available data material (Eftestøl et al., 2000). For all registered defibrillations, the corresponding VF waveform segment immediately prior to a defibrillation attempt was extracted and grouped according to the corresponding outcome being ROSC or NO ROSC as illustrated in Figure 2. For each segment, the PSD was estimated. From the PSD, four spectral features: the centroid frequency, peak power frequency, spectral flatness and energy were computed (Eftestøl et al., 2000). From a feature set being a decorrelated representation of the original spectral feature set, the two most expressive features, $\mathbf{V}_{\mathrm{PCA}_{1}}$ and $\mathrm{V}_{\mathrm{PCA}_{2}}$, yielded the highest performing outcome predictor (Eftestøl et al., 2000). Figure 3 illustrates how these two feature components

ROSC

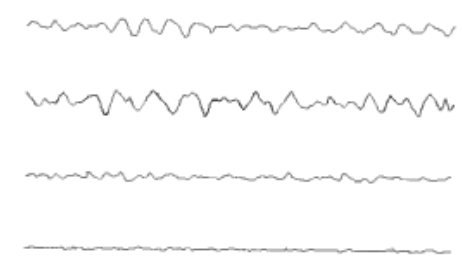

NO ROSC

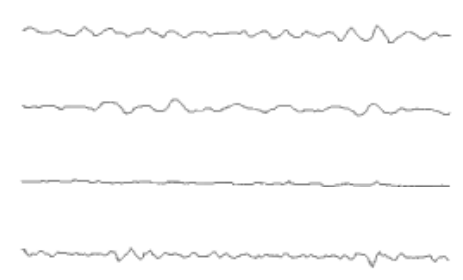

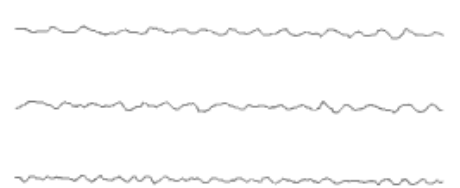

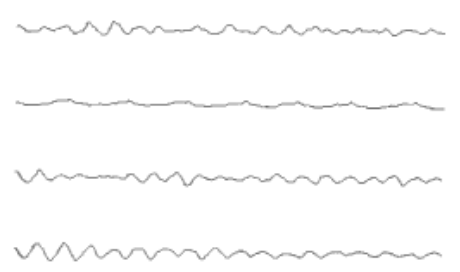

Figure 2: Examples of VF waveforms extracts from immediately prior to defibrillations. Each tracing corresponds to four seconds of ECG tracing. The four second tracings immediately prior to the two first defibrillations in Figure 1 would fall in the unsuccessful group labeled NO ROSC, while the four seconds of tracing immediately prior to the third defibrillation would fall in the successful group labeled ROSC. The different size of the two groups illustrates the typically higher a priori probability of the NO ROSC class. 




Figure 3: The feature representations of all the VF waveforms extracts shown as coordinates in feature space. For each of the 87 ROSC $(*)$ and 781 NO ROSC $(\bullet)$ extracts the Fourier transform was computed to estimate the Power spectral density (PSD). From the PSD four spectral features, the centroid frequency, peak power frequency, spectral flatness and the energy feature were computed. These four features were further reduced into two expressive features, $v_{\mathrm{PCA}_{1}}$ and $\mathrm{v}_{\mathrm{PCA}_{2}}$, through decorrelation. These two features yields the highest performing defibrillation outcome predictor. Each of the VF waveform extracts shown in Figure 2 is represented as a coordinate $\left(\mathrm{V}_{\mathrm{PCA}_{1}}, \mathrm{v}_{\mathrm{PCA}_{2}}\right)$ in this feature space.

measured from $87 \operatorname{ROSC}(*)$ and 781 NO ROSC $(\bullet)$ VF segments distribute as coordinates in feature space.

In a related study, the á posteriori probability function for the ROSC class was used to represent the probability of successful outcome, $P_{\text {ROSC, }}$, as shown in Figure 4 (Eftest $\varnothing 1$ et al., 2001). The ECG features and the $P_{\mathrm{R} O S C}$ function can be computed online, offering a tool for monitoring the patient's resuscitability during therapy. The predictive value of any such $P_{\text {RosC }}$ must be established. In Eftestøl et al., 2000 we did this by investigating the $P_{\text {Rosc }}$-features' ability to predict defibrillation outconne. Recently we applied the decision rules established in the original study (Eftestøl et al., 2000) on new data. The results showed good correspondence between the training and independent test results (Eftestøl et al., 2005).

We investigated the monitoring capabilities of $P_{R O S C}$ in two studies. In one study we used $\mathrm{P}_{\mathrm{R} O \mathrm{SC}}$ to evaluate the effect of interrupting precordial compressions (Eftestøl et al., 2002) and in more recent study we investigated the effects of giving precordial compressions (Eftestøl et al., 2004). In conclusion, the studies demonstrated a negative change in $\mathrm{P}_{\mathrm{ROSC}}$ due to interrupting compressions (Eftestøl et al.. 2002) and a positive change after prolonged sequences of precordial compressions (Eftestøl et al., 2004).

Other groups have investigated features, individually, or combined into indicators for resuscitability similar to $\mathrm{P}_{\mathrm{ROSC}}$, using regression techniques or pattern recognition 


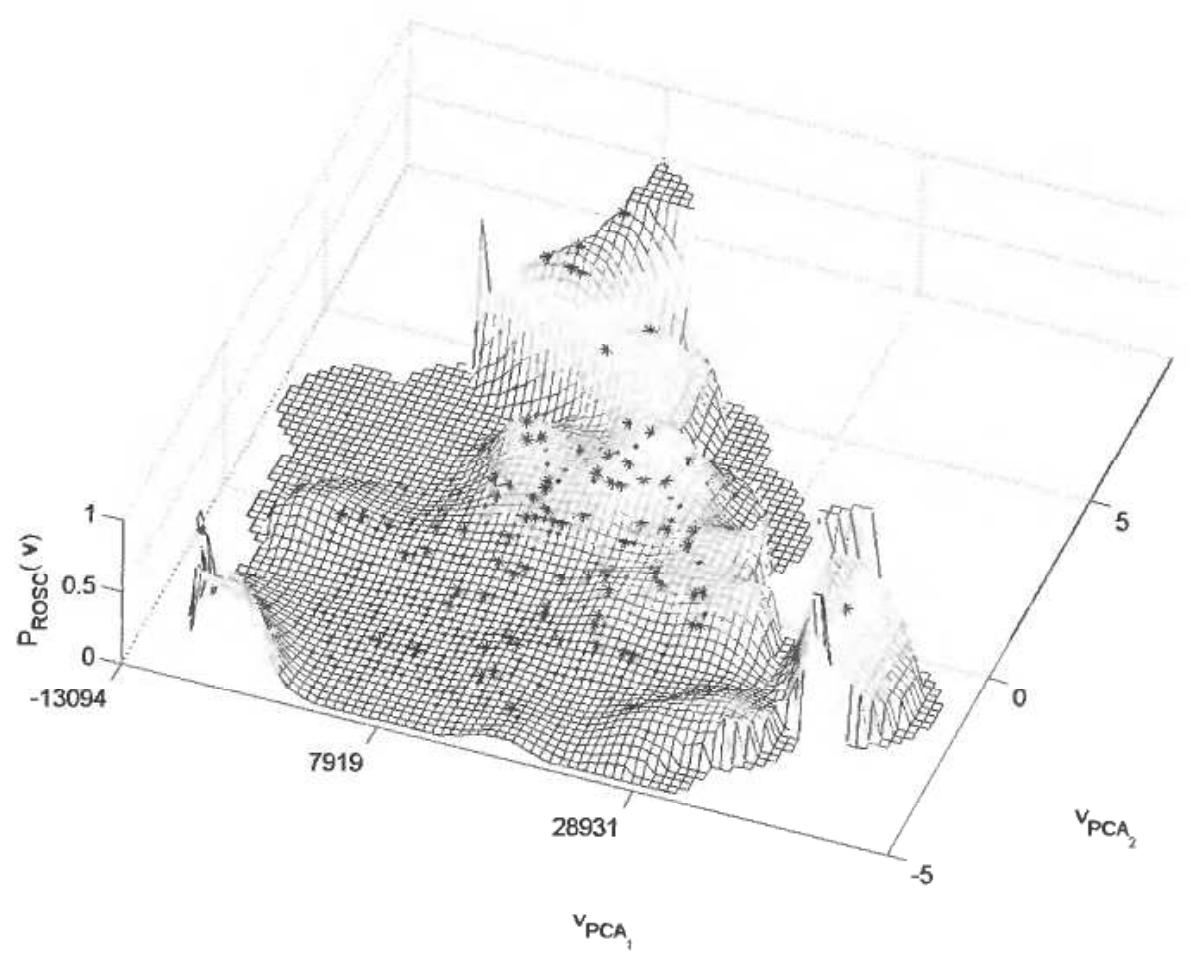

Figure 4: A functional relationship between the feature representations of all the VF waveforms extracts and corresponding ROSC/NO ROSC outcome with the feature representation coordinates from Figure 3 superimposed. This can be explained as a division of the feature space defined by $\mathrm{V}_{\mathrm{PCA}_{\mathrm{E}}}$ and $\mathrm{V}_{\mathrm{PCA}_{2}}$ into equal-sized cells. In each cell the $\mathrm{P}_{\mathrm{ROSC}}$ value is estimated as the number of ROSC coordinates divided by the total number of coordinates in the cell.

methods (Noc et al., 1999; Amann et al., 2001; Achleitner et al., 2000; Watson et al., 2000; Monsieurs et al., 1998). When used in animal studies, both outcome prediction and correlation analysis between the features and established indicators of resuscitability have been applied for feature evaluation. One of the problems with evaluating features in animal studies is that features with high discriminative power in animal studies often perform much worse when applied to human data, possibly due to the differences in VF frequency spectra between experimental animals and patients.

\section{Reducing duration of hands-off-intervals}

AEDs record and analyse the ECG in order to make a shock/no-shock decision. However, the mechanical activity from chest compressions and ventilations during CPR introduces artefact components in the ECG (Langhelle et al., 2001). For AEDs to perform reliable ECG signal analysis, CPR is therefore discontinued for a substantial time before the potential delivery of an electric shock. Reduction or elimination of this "hands-off" time requirement by removing these artefacts, should significantly improve defibrillation success rate (Sato et al., 1997; Yu et al., 2002).

The artefacts have been successfully removed from animal ECGs by applying high-pass digital filters with fixed coefficients (Noc et al., 1999; Strohmenger et al., 


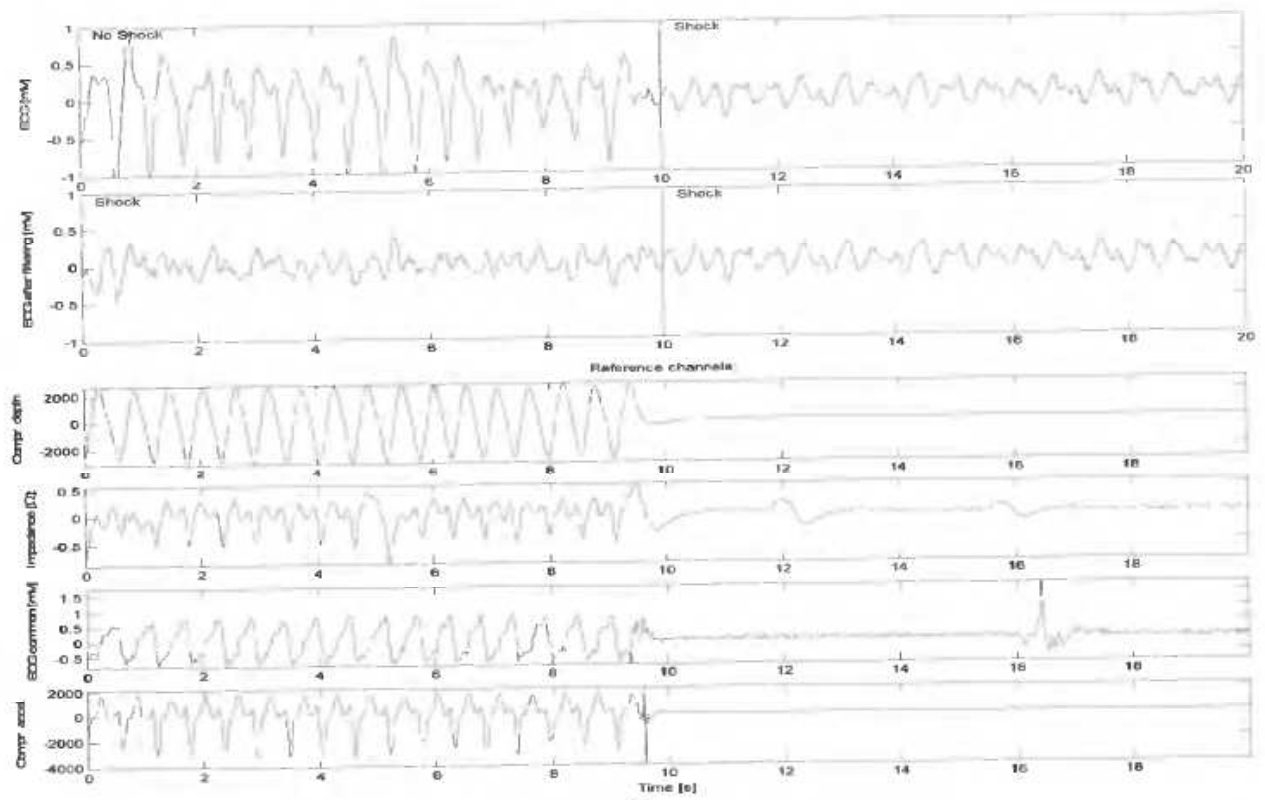

(a)


(b)

Figure 5: Two examples of CPR artefact removal using MC-RAMP: Shockable rhythm: VF segment (a), Non-shockable rhythm: PEA segment (b). Each segment is 20 sec. with CPR on first half. Each half is presented to a shock/no-shock classifier with the shock advice written on the plot. From top to bottom the plots are ECG before and after filtering, and four reference signals; compression depth, thorax impedance, ECG common, and compression acceleration, respectively. 


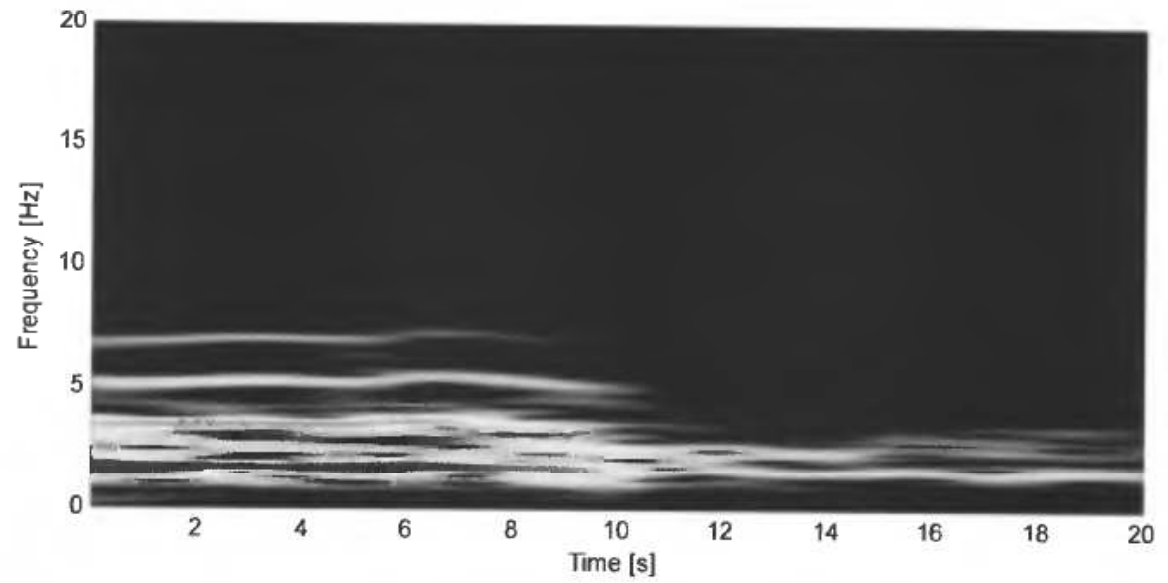

(a)

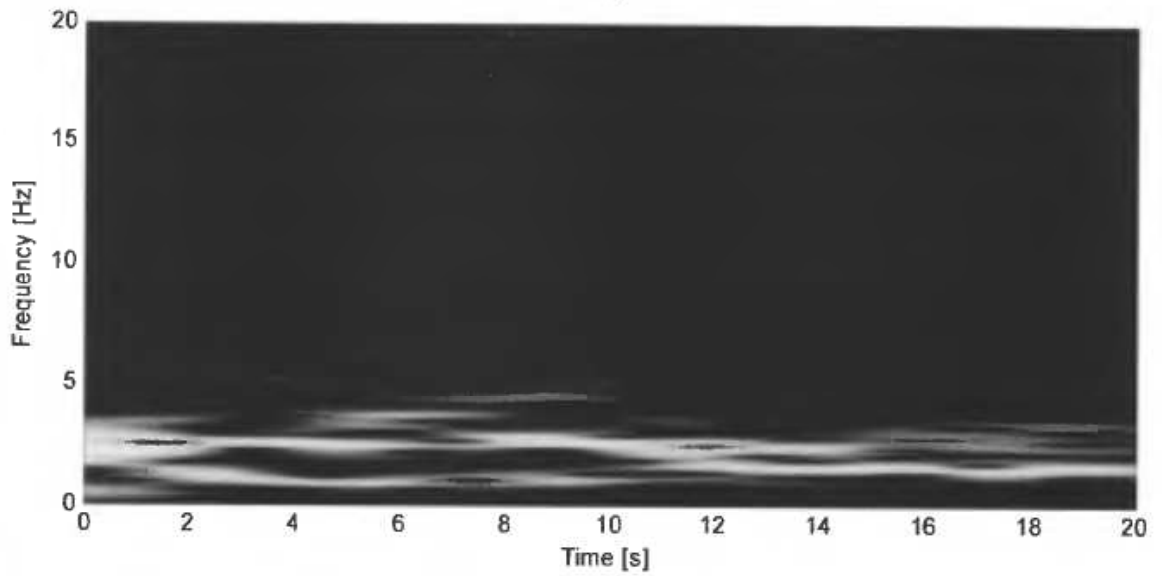

(b)

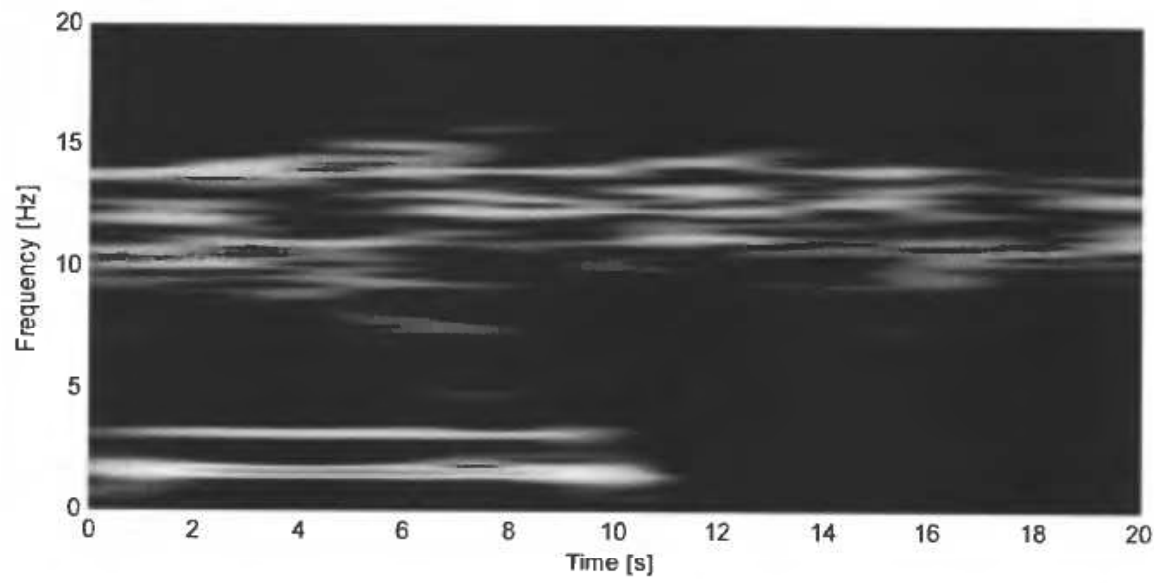

(c)

Figure 6: Spectrograms (time-frequency plots where each vertical line can be said to be the PSD for a given time instant) for ECG with CPR artefacts on the first 10 seconds. (a) Spectrogram of the VF signal from Figure 5(a). (b) Spectrogram of the VF signal from Figure 5(a) after filtering of CPR artefacts using MC-RAMP. (c) Spectrogram of CPR in pig VF showing non-overlapping frequency components of pig VF and CPR 
1996). In human ECG, however, the frequency components of the artefacts overlap with the frequency components of the desired signal, which makes separation by such filters infeasible.

In a mix of pig CPR artefacts and human VF/VT, CPR artefacts were successfully reduced using a multichannel time varying Wiener filter (Aase et al., 2000) and a multichannel recursive adaptive matching pursuit (MC-RAMP) filter (Husoy et al., 2002). These types of filters use reference signals that correlate with/resemble different artefact types, e.g. compressions and ventilations, and are recorded simultaneously with the ECG. Using these signals, the CPR artefacts are modelled recursively in the filter and subtracted from the ECG, revealing the underlying heart rhythm.

Two examples of filtering using MC-RAMP are shown in Figure 5. The spectrograms of the VF signal in Figure 5(a) before and after filtering are shown in Figure 6(a) and (b), respectively. As seen from these plots, the frequency components of the CPR and human VF overlap, making the filtering non-trivial. For comparison, Figure 6(c) shows the spectrogram of CPR in pig VF, showing non-overlapping frequency components of VF and CPR artefacts making such a signal trivial to filter using standard frequency-selective filters.

Recently we have worked on using the MC-RAMP algorithm for realistic removal of CPR artefacts in human out-of-hospital cardiac arrest ECG (Eilevstjønn et al., 2004). While CPR artefacts in the ECG can be reduced using MC-RAMP, it may not always be good enough for the shock/no-shock decision classifier. In a recent study, we have analysed and quantified the benefit of using artefact removal during CPR (Eilevstjønn $e t$ $a l ., 2005)$. The study concluded that the time without blood flow from compressions can be reduced significantly, hopefully increasing the survival (Eilevstjønn et al., 2005).

Optimising CPR quality using thorax impedance based measurements of ventilation and pulse

An animal study implies the application of transthoracic impedance signals for breath- and pulse-check (Pellis et al., 2002). Recent experiments have demonstrated the potential of measuring ventilation and pulse using thoracic impedance on realistic human cardiac arrest data (Risdal et al., 2005) (ris1, ris2, ris3, ris4).

In (Risdal et al., 2005) (ris1) we studied the relationship between lung volume changes and resulting thoracic impedance changes measured by a modified defibrillator to evaluate the potential of using thoracic impedance for estimation of ventilation rate, inspiration time and tidal volume. The respiratory and impedance waveform of a ventilation cycle was found to be highly correlated. This facilitates ventilation rate and inspiration time estimation in a CPR quality feedback system, but the results also showed that thoracic impedance is not suitable for estimation of tidal volume. A pattern classifier did not perform convincingly in discriminating between to low, sufficient and to much ventilation (Risdal et al., 2005) (ris2).

In Risdal et al., 2005 (ris3) we investigated the potential of acquiring circulatory information from patients undergoing resuscitation. We studied the correlation between impedance-derived parameters related to circulation and arterial blood pressure measurements in 58 patients. The results showed poor correlation between impedance and pressure measurements indicating that the impedance-derived parameters are not suitable for quantification of circulation but can be used to indicate circulation. In Risdal et al., 2005 (ris4) a neural network-based classifier using ECG and thorax impedance recorded 
by AEDs to distinguish between pulse rhythms and pulseless electrical activity demonstrated the possibility of indicating circulation automatically.

\section{Conclusion}

Defibrillation is the single most important decisive factor for outcome from out-ofhospital cardiac arrest. Recent research has identified and renewed focus on the important role of chest compressions and ventilation. Retrospective analyses of information stored electronically during therapy have offered additional explanations for the weak links in the chain of survival. Signal analysis and modelling of cardiac arrest ECG for prediction of shock outcome and monitoring of resuscitability have been applied in diverse ways with gradual use of more sophisticated methods in recent years. Focus has also turned on other factors, modelling compressions, ventilations and automated pulse detection. The studies so far are retrospective and indicate a potential that these methods have a potential for monitoring resuscitability during therapy.

We believe that collection of large amounts of data allowing more accurate and general models might fuse these methods into a decision support system for cardiopulmonary resuscitation, suitable to be integrated into AEDs within the next three years. Thus, development of advanced digital signal processing algorithms for decision support systems might provide online guidance to emergency medicine personnel equipped with an AED. Based on this guidance, the various components of ALS can be administered optimally. Thus, a sequence of treatment steps can be given based on the estimated state of each individual patient, rather than according to some specified fixed protocol as is common today. This is expected to significantly increase the survival rate.

\section{References}

AASE, S. O., EFTESTøL, T. \& HuSøY, J. H. (2000). CPK artifact removal from human ECG using optimal multichannel filtering. IEEE Trans BME, 48, pp. 1440-1449.

Achleitner, U., Wenzel, V. \& Strohmenger, H. U. (2000). The effects of repeated doses of epinephrine on ventricular fibrillation in a porcine model of prolonged cardiopulmonary resuscitation. Anesth Analg, 90, pp. 1067-1075.

Amann, A., Rheinberger, K. \& ACHLeitner, U. (2001). Algorithms to analyze ventricular fibrillation signals. Current Opinion in Critical Care, 7, pp. 152156.

CobB, L. A., Fahrenbruch, C. E. \& Walsh,T. R. (1999). Influence of cardiopulmonary resuscitation prior to defibrillation in patients with out-of-hospital ventricular fibrillation. JAMA, 281(13), pp. 1181-1188.

Cummins, R. O., Eisenberg, M. S., Hallstrom, A. P., Hearne, T. R., Graves, J. R. \& Litwin, P. E. (1985) What is a "save"?: Outcome measures in clinical evaluations of autmatic external defibrillators. American Heart Journal, 110, pp. 1133-1138.

DudA, R. O., HART, P. E. \& STORK, D. G. (2001). Pattern classification: Second edition. John Wiley and Sons, New York (NY).

Eftestøl, T., EilevstjønN, J. \& STEen, P. A. (2003). Advanced life support therapy on out-of-hospital cardiac arrest patients: an engineering perspective. Expert Rev. Cardiovasc. Ther., 1(2), pp. 89-99.

Eftestøl, T., Losert, H., Kramer-Johansen, J., Wik, L., Sterz, F. \& Steen, P. A. (2005). Independent evaluationof a defibrillation outcome predictor for out-of-hospital cardiac arrest patients. Resuscitation, In press.

EFtestøl, T., Sunde, K. \& AASE, S. O. (2001). 'Probability of successful defibrillation' as a monitor during CPR in out-of-hospital cardiac arrested patients. Resuscitation, 48, pp. 245-254.

EFtestøl, T., Sunde, K. \& AASE, S. O. (2000). Predicting outcome of defibrillation by spectral characterization and nonparametric classification of ventricular fibrillation in patients with out-of-hospital cardiac arrest. Circulation, 102, pp. 1523-1529. 
EfTestøl, T., Sunde, K. \& STEEN, P. A. (2002). The effects of interrupting precordial compressions on the calculated probability of defibrillation success during out-of-hospital cardiac arrest. Circulation, 105, pp. 2270-2273.

EFTESTøL, T., WIK, L., SUNDE, K. \& STEEN, P. A. (2004). Effects of cardiopulmonary resuscitation on predictors of ventricular fibrillation defibrillation success during out-of-hospital cardiac arrest. Circulation, 110, 10-15.

Eilevstiøn,, J., Eftestøl, T., Aase, S. O., Myklebust, H., Husøy, J. H. H. \& Steen, P. A. (2004). Feasability of shock advice analysis during CPRthrough removal of CPR artefacts from human ECG. Resuscitation, 2, pp. 131-141.

Eilevstjønn, J., Kramer-Johansen, J., Eftestøl, T., Stavland, M., Myklebust, H. \& Steen, P. A. (2005). Reducing no flow times during automated external defibrillation. Resuscitation In press.

EisenberG, M. S., Horwood, B. T. \& Cummins, R. O. (1990). Cardiac arrest and resuscitation: A tale of 29 cities. Ann Emerg Med, 19, pp. 179-186.

Fong, A. C. M., HU, S. C. \& JHA, G. (2002). Data mining for decision support. IT Pro March/April, pp. 9-17.

GLINER, B. E. \& WHITE, R. D. (1999). Electrocardiographic evaluation of defibrillation shocks delivered to out-of-hospital sudden cardiac arrest patients. Resuscitation, 41, pp. 133-144.

Guidelines 2000 for cardiopulmonary resuscitation and emergency cardiovascular care. Part 4: The automated external defibrillator. Circulation 102(suppl I), I-60-I-76 (2000).

Hargarten, K. M., Stueven, H. A. \& Waite, E. M. (1990). Prehospital experience with defibrillation of coarse ventricular fibrillation: a ten-year review. Ann Emerg Med, 19, pp. 157-162.

HerlitZ, J., BAHR, J. \& FiSCHER, M. (1999). Resuscitation in Europe: a tale of five European regions. Resuscitation, 41, pp. 121-131.

Husøy, J. H., Ellevstjønn, J., Eftestøl, T., Aase, S.O., Myklebust, H. \& Steen. P. A. (2002). Removal of cardiopulmonary resuscitation artifacts from human ECG using an efficient matching pursuit-like algorithm. IEEE Trans BME, 49(11), pp. 1287-1298.

KovalerchuK, B., Vityaev, E. \& Ruiz, J. F. (2000). Consistent knowledge discovery in medical diagnosis. IEEE Engineering in Medicine and Biology Magazine, 19(4), pp. 26-37.

LANGHelle, A., EFTestøl, T. \& MyKLebust, H. (2001). Reducing CPR artefacts in ventricular fibrillation in vitro. Resuscitation, 48, pp. 279-291.

Larsen, M. P., Eisenberg, M. S., Cummins, R. O. \& Hallstrom, A. P. (1993). Predicting survival from out-of-hospital cardiac arrest: a graphic model. Ann Emerg Med, 22, pp. 1652-1658. 1999;281:1182-1188

Marenco, J. P., WANG, P. J., Link, M. S., Homud, M. K. \& Estes, N. A. M. (2001). Improving survival from sudden cardiac arrest: The role of the automated defibrillator. JAMA, 285(9), pp. 1193-1200.

Mrtra, S., Pal, S. K. \& Mrtra, P. (2002). Data mining in soft computing framework: A survey. IEEE Transactions on Neural Networks, 13(1), pp. 3-14.

Monsieurs, K. G., De Cauwer, H. \& WuYTS, F. L. (1998). A rule for early outcome classification of out-of-hospital cardiac arrest patients presenting with ventricular fibrillation. Resuscitation 36, pp. 37-44.

Noc, M., WeILl, M. H. \& TANG, W. (1999). Electrocardiographic prediction of the success of cardiac resuscitation. Crit Care Med, 27(4), pp. 708-714.

Peluis, T., Bisera, J., TANG, W. \& Weil, M. H. (2002). Expanding automatic external defibrillators to include automated detection of cardiac, respiratory, and cardiorespiratory arrest. Crit Care Med, 30(4), pp. S176-S178.

Povoas, H. P., Weil, M. H., Tang, W., Bisera, J., Klouche, K. \& Barbatis, A. (2002). Predicting the success of defibrillation by electrocardiographic analysis. Resuscitation, 53, pp. $77-82$.

Risdal, M., Losert, H., Nys/eter, J., Eftestøl, T., Sterz, F. \& Aase, S. O. (ris3) (in press). Examining the potential of using thorax impedance measured by automated external defibrillators for quantification of circulation. In Proc. Computers in Cardiology '05, Lyon. France, September 2005.

Risdal, M., Losert, H., Nyseter, J., Eftestøl, T., Sterz, F. \& Aase, S. O. (ris1) (in press). Non-invasive ventilation monitoring during cardipulmonary resuscitation by means of thoracic impedance. In Proc. $3^{\text {rd }}$ European Medical and Biological Engineering Conference '05, Prague, Czech Repeublic, November 2005. 
Risdal, M., Losert, H., Nyseter, J., Eftestøl, T., Sterz, F. \& Aase, S. O. (ris2) (in press). Ventilation classification for cardiopulmonary resuscitation quality monitoring using thoracic impedance. In Proc. $3^{\text {rd }}$ European Medical and Biological Engineering Conference '05, Prague, Czech Repeublic, November 2005.

Risdal, M., Sleveland, T., Steen, P. A., Kramer-Johansen, J., Wik, L, Sterz, F., Losert, H., EFTESTøL, T. \& AASE, S. O. (ris4) (in press). Improving rhythm classification using electrocardiogram and thorax impedance recorded by automated external defibrillators. In Proc. NORSIG '05, Stavanger, Norway, September 2005.

SATO, Y., WEIL, M. H. \& SUN, S, (1997). Adverse effects of interrupting precordial compression during cardiopulmonary resuscitation. Crit Care Med, 25, pp. 733-736.

Small, M., YU, D. J. \& Clayton, R. (2001). Temporal evolution of nonlinear dynamics in ventricular arrhythmia. International Journal of Bifurcations and Chaos, 11(10), pp. 25312548.

StrohmenGer, H. U., EFTEStøl, T. \& Sunde, K. (2001). Predictive value of ventricular fibrillation ECG signal frequency and amplitude parameters in patients with out-of-hospital cardiac arrest. Anasthesia and Analgesia, 93, pp. 1428-1433.

Strohmenger, H. U., Lindnder, K. H., Lindner, I. M., Pfenniger, E. G. \& Bothner, U. (1996). Spectral analysis of ventricular fibrillation and closed-chest cardiopulmonary resuscitation. Resuscitation, 33(2), pp. 155-161.

Sunde, K., EFtestøl, T. \& AschenberG. C. (1999). Quality assessment of detibrillation and ALS using data from the medical control module of the defibrillator. Resuscitation 41, pp. $237-$ 247.

Sunde, K., Fremstad, K. O., Furuheim, J. \& Steen, P. A. (2001). Ambulance response intervals in connection with cardiac arrest in Oslo. Tidsskr Nor Lageforen, 121, pp. 900-903.

VAN Hoeywegen, R. J., Bossaert, L. L. \& Mullie, A. (1993). Belgian cerebral resuscitation study group. Quality and efficiency of bystander CPR. Resuscitation, 26, pp. 47-52.

VÄrri, A., KemP, B.. PEnZEL, T. \& SClöGl, A. (2001). Standards for biomedical signal databases. IEEE Engineering in Medicine and Biology Magazine, 20(3), pp. 33-37.

WATSON, J. N., AdDison, P. S. \& ClEGG, G. R. (2000). A novel wavelet transform based analysis reveals hidden structure in ventricular fibrillation. Resuscitation, 43, pp. 121-127.

WiK, L., Brennan, R. T. \& BRaslow, A. (1995). A peer-training model for instruction of basic life support. Resuscitation. 29, pp. 119-128.

WIK, L., HANSEN, T. B. \& FYLLING, F. (2003). Delaying defibrillation to give basic cardiopulmonary resuscitation to patients with out-of-hospital ventricular fibrillation: A randomized trial. JAMA, 289(11), pp. 1389-1395.

YU, T., WEIL, M. H. \& TANG, W. (2002). Adverse outcomes of interupted precordial compression during automated defibrillation. Circulation. 106, pp. 368-372. 